Honam Mathematical J. 35 (2013), No. 1, pp. 037-049

http://dx.doi.org/10.5831/HMJ.2013.35.1.37

\title{
A CHARACTERIZATION OF ELLIPTIC HYPERBOLOIDS
}

\author{
Dong-Soo KIM* And Booseon Song
}

\begin{abstract}
Consider a non-degenerate open convex cone $C$ with vertex the origin in the $n$-dimensional Euclidean space $\mathbb{E}^{n}$. We study volume properties of strictly convex hypersurfaces in the cone $C$. As a result, for example, if the volume of the region of an elliptic cone $C$ cut off by the tangent hyperplane $P$ of $M$ at $p$ is independent of the point $p \in M$, then it is shown that the hypersurface $M$ is part of an elliptic hyperboloid.
\end{abstract}

\section{Introduction}

We will say that a convex hypersurface in the $n$-dimensional Euclidean space $\mathbb{E}^{n}$ is strictly convex if the hypersurface is of positive GaussKronecker curvature $K$ with respect to the unit normal $N$ pointing to the convex side.

Let $C$ denote a non-degenerate open convex cone with vertex the origin. Suppose that $M \subset C$ is a smooth strictly convex hypersurface in the $n$-dimensional Euclidean space $\mathbb{E}^{n}$ with the unit normal $N$ pointing to the convex side. For a point $p \in M$, we denote by $V(p)$ and $A(p)$ the volume of the region of the cone $C$ cut off by the tangent hyperplane $P$ of $M$ at $p$ and the $(n-1)$-dimensional area of the section $P \cap C$ in the hyperplane $P$, respectively.

Recently, the first author and Y. H. Kim studied some volume properties of strictly convex hypersurfaces in the $n$-dimensional Euclidean space $\mathbb{E}^{n}$ and gave some characterizations of hyperspheres and elliptic

Received December 18, 2012. Accepted January 21, 2013.

2010 Mathematics Subject Classification. 53A07.

Key words and phrases. strictly convex hypersurface, elliptic hyperboloid, homogeneous extension, $n$-dimensional volume, elliptic cone, support function.

${ }^{*}$ Corresponding author 
paraboloids $([2,3])$. Using volume properties of strictly convex hypersurfaces, the first author also established some characterizations of ellipsoids and elliptic hyperboloids in the Euclidean space $\mathbb{E}^{n}([4])$.

In this paper, we consider a non-degenerate open convex cone $C$ with vertex the origin and study strictly convex hypersurfaces $M$ in the cone $C$ which satisfy the following conditions.

$(V): V(p)$ is a positive constant on $M$.

The area function $A(p)$ can be given by the volume function $V(p)$ as follows.

If we denote by $h(p)$ the support function of $M$ at $p$ with respect to the origin $([1,5])$, which is defined by $h(p)=\langle p, N(p)\rangle$, then we get

$$
n V(p)=|h(p)| A(p) .
$$

Suppose that a strictly convex hypersurface $M$ in the cone $C$ is given by $M=g^{-1}(k)$ for some smooth function $g: U \subset C \rightarrow \mathbb{R}$. Then, for a nonzero constant $d$ we introduce the homogeneous extension $g_{d}$ of $g$ (or of $M)$ as follows.

$$
g_{d}(t p)=k t^{d}, \quad p \in M,
$$

where it is defined. The homogeneous extension $g_{d}$ is well-defined on an open set of $\mathbb{E}^{n}$ containing a point $p_{0} \in M$ where the tangent hyperplane of $M$ does not pass through the origin. Then the homogeneous extension $g_{d}$ of $g$ is a homogeneous function of degree $d$ and the hypersurface $M$ is a level hypersurface $g_{d}^{-1}(k)$ of $g_{d}$. Note that in case $g$ is homogeneous of degree $d$, then the homogeneous extension $g_{d}$ of the function $g$ is the same as the function $g$.

Now, we use the unit normal $N(p)= \pm \nabla g_{d}(p) /\left|\nabla g_{d}(p)\right|$ at $p \in M$, where $\nabla g_{d}(p)$ denotes the gradient of $g_{d}$ at $p \in M$. Then the height function $h(p)$ satisfies

$$
h(p)\left|\nabla g_{d}(p)\right|= \pm d k .
$$

Thus, it follows from (1.1) that the volume function $V(p)$ and the area function $A(p)$ are related by

$$
V(p)=\frac{|d k|}{n} \frac{A(p)}{\left|\nabla g_{d}(p)\right|} .
$$


Therefore, it follows from (1.4) that for a strictly convex hypersurface $M=g^{-1}(k)$ in the cone $C$, the condition $(V)$ is equivalent to the following condition:

$(A)$ : For some nonzero constant $d, A(p) /\left|\nabla g_{d}(p)\right|$ is a positive constant on $M$, where $\nabla g_{d}(p)$ denotes the gradient of $g_{d}$ at $p \in M$.

As a result, we establish some characterizations of hypersurfaces in a non-degenerate convex cone $C$ as follows.

First, in Section 2, we consider the convex cone $C:\left\langle x, v_{i}\right\rangle>0, i=$ $1, \cdots, n$ surrounded by hyperplanes $P_{i}:\left\langle x, v_{i}\right\rangle=0$. Here, $v_{1}, \cdots, v_{n}$ denote the linearly independent $n$ unit vectors in the $n$-dimensional Euclidean space $\mathbb{E}^{n}$. In this case, we get

Theorem 2.3. Suppose that a strictly convex hypersurface $M$ in the $n$-dimensional Euclidean space $\mathbb{E}^{n}$ lies in the above cone $C$. Then, the following are equivalent.

1) The volume $V(p)$ is a positive constant on $M$.

2) The hypersurface $M$ is part of a component of a level hypersurface $g^{-1}(c)$ with $c>0$ of the homogeneous polynomial $g(x)=\prod_{i=1}^{n}\left\langle x, v_{i}\right\rangle$.

Next, in Section 3, we consider the elliptic cone $C: x_{n}^{2}>a_{1}^{2} x_{1}^{2}+\cdots+$ $a_{n-1}^{2} x_{n-1}^{2}, x_{n}>0$, where $a_{i}>0$ for $i=1, \cdots, n-1$. In this case, we prove

Theorem 3.4. Suppose that a strictly convex hypersurface $M$ in the $n$ dimensional Euclidean space $\mathbb{E}^{n}$ lies in the above elliptic cone $C$. Then, the following are equivalent.

1) The volume $V(p)$ is a positive constant on $M$.

2) The hypersurface $M$ is part of an elliptic hyperboloid $g^{-1}(c)$ with $c<0$ which is defined by

$$
g\left(x_{1}, x_{2}, \cdots, x_{n}\right)=a_{1}^{2} x_{1}^{2}+\cdots+a_{n-1}^{2} x_{n-1}^{2}-x_{n}^{2}, \quad x_{n}>0 .
$$

Throughout this article, all objects are smooth and connected, unless otherwise mentioned. 


\section{Cones surrounded by linearly independent hyperplanes}

In this section, first of all, we consider the cone $C: x_{i}>0, i=1, \cdots, n$ surrounded by the coordinate hyperplanes $P_{i}: x_{i}=0, i=1, \cdots, n$.

Suppose that a smooth strictly convex hypersurface $M$ in the $n$ dimensional Euclidean space $\mathbb{E}^{n}$ with a nonzero normal $Z$ lies in the cone $C: x_{i}>0, i=1, \cdots, n$.

Lemma 2.1. If we let $Z(p)=\left(Z_{1}(p), \cdots, Z_{n}(p)\right)$, then the volume $V(p)$ is given by

$$
V(p)=\frac{ \pm\langle p, Z(p)\rangle^{n}}{n ! Z_{1}(p) \cdots Z_{n}(p)},
$$

where the denominator does not vanish.

Proof. The tangent hyperplane $P$ of $M$ at $p$ is given by

$$
\langle x, Z(p)\rangle=\langle p, Z(p)\rangle .
$$

Hence, for each $i=1, \cdots, n$ the hyperplane $P$ meets the $x_{i}$-axis at $x_{i}=\langle p, Z(p)\rangle / Z_{i}(p)$, if $Z_{i}(p) \neq 0$. This completes the proof.

Proposition 2.2. Suppose that a smooth strictly convex hypersurface $M$ in the $n$-dimensional Euclidean space $\mathbb{E}^{n}$ lies in the cone $C: x_{i}>$ $0, i=1, \cdots, n$. Then the following are equivalent.

1) The volume $V(p)$ is a positive constant on $M$.

2) The hypersurface $M$ is part of a component of a level hypersurface $g^{-1}(c)$ with $c>0$ of the homogeneous polynomial $g\left(x_{1}, \cdots, x_{n}\right)=$ $x_{1} \cdots x_{n}$.

Proof. The hypersurface $M$ is, locally, the graph of a function. Hence, for a fixed point $p_{0} \in M$, we may assume that a neighborhood of $p_{0} \in M$ is the graph of a function $x_{n}=f(x), x=\left(x_{1}, \cdots, x_{n-1}\right)$ defined on an open set $O$ in the Euclidean space $\mathbb{E}^{n-1}$.

We consider the nonzero normal $Z(p)$ to $M$ at $p=(x, f(x))$ given by

$$
Z(p)=\left(-f_{1}(x), \cdots,-f_{n-1}(x), 1\right),
$$

where $f_{i}$ denotes the partial derivative of $f$ with respect to $x_{i}, i=$ $1, \cdots, n-1$. Then, Lemma 2.1 shows that for the constant $a= \pm(n !)$ the volume $V(p)$ is given by

$$
a V(p)=\{\langle x, \nabla f(x)\rangle-f(x)\}^{n} W(x)^{-1},
$$

where $W(x)=\prod_{i=1}^{n-1} f_{i}(x)$ does not vanish. 
Henceforth, we proceed on the open set $O_{1} \subset O$ where $W(x) \neq 0$. By differentiating (2.4) with respect to $x_{i}, i=1, \cdots, n-1$, we have for each $i=1, \cdots, n-1$

$$
\frac{\partial}{\partial x_{i}}\{a V(p)\}=W^{-1}\{\langle x, \nabla f(x)\rangle-f(x)\}^{n-1} \sum_{j=1}^{n-1} f_{i j} \frac{A_{j}}{f_{j}},
$$

where $A_{j}$ is defined by for $j=1,2, \cdots, n-1$

$$
A_{j}(x)=n x_{j} f_{j}(x)-\langle x, \nabla f(x)\rangle+f(x) .
$$

Now, we suppose that the volume function $V(p)$ is a positive constant on $M$. Then, it follows from (2.5) that on the open set $O_{1}$ we have

$$
\{\langle x, \nabla f(x)\rangle-f(x)\}^{n-1} \sum_{j=1}^{n-1} f_{i j} \frac{A_{j}}{f_{j}}=0
$$

for each $i=1,2, \cdots, n-1$. It follows from (2.4) that $\langle x, \nabla f(x)\rangle-f(x) \neq$ 0 on the open set $O_{1}$. Hence (2.7) implies that on the open set $O_{1}$ we obtain

$$
\sum_{j=1}^{n-1} f_{i j}(x) \frac{A_{j}(x)}{f_{j}(x)}=0
$$

for each $i=1,2, \cdots, n-1$.

Since $M$ is strictly convex, $\operatorname{det}\left(f_{i j}(x)\right)$ is nonzero ([6, p.93]). Together with (2.8), this shows that on the open set $O_{1}$, each $A_{j}(x)$ vanishes. Hence, (2.6) yields that for $j=1,2, \cdots, n-1$

$$
n x_{j} f_{j}(x)=\langle x, \nabla f(x)\rangle-f(x) .
$$

Thus, we have

$$
x_{j} f_{j}(x)=x_{k} f_{k}(x), \quad j, k=1,2, \cdots, n-1,
$$

and hence again we get $x_{j} f_{j}(x)+f(x)=0$ for all $j=1,2, \cdots, n-1$.

This implies that the function $h(x)=x_{1} \cdots x_{n-1} f(x)$ is a nonzero constant. That is, for some nonzero constant $c, f(x)=c\left(x_{1} \cdots x_{n-1}\right)^{-1}$ on the open set

$$
O_{1}=\left\{x \in O \mid f_{1}(x) \cdots f_{n}(x) \neq 0\right\} .
$$

But, the function $f(x)=c\left(x_{1} \cdots x_{n-1}\right)^{-1}$ satisfies $f_{1}(x) \cdots f_{n}(x) \neq 0$ where it is defined. This shows that $O_{1}=O$ and hence every point on the neighborhood of $p_{0}$ satisfies $x_{1} \cdots x_{n}=c$. 
By connectedness of $M$, we conclude that $M$ is part of a component of a level hypersurface $g^{-1}(c)$ with $c>0$ of the function $g\left(x_{1}, \cdots, x_{n}\right)=$ $x_{1} x_{2} \cdots x_{n}$.

Conversely, suppose that $M$ is a component of a level hypersurface $g^{-1}(c), c>0$ of the function $g\left(x_{1}, \cdots, x_{n}\right)=x_{1} x_{2} \cdots x_{n}$. Then, using Lemma 2.1 with $Z=\nabla g$, we get $V(p)=\frac{n^{n}}{n !} c$.

This completes the proof.

Now, let's denote by $v_{1}, \cdots, v_{n}$ the linearly independent $n$ unit vectors in the $n$-dimensional Euclidean space $\mathbb{E}^{n}$. We consider the cone $C:\left\langle x, v_{i}\right\rangle>0, i=1, \cdots, n$ surrounded by hyperplanes $P_{i}:\left\langle x, v_{i}\right\rangle=0$.

Theorem 2.3. Suppose that a strictly convex hypersurface $M$ lies in the cone $C$. Then the following are equivalent.

1) The volume $V(p)$ is a positive constant on $M$.

2) The hypersurface $M$ is part of a component of a level hypersurface $g^{-1}(c)$ with $c>0$ of the homogeneous polynomial $g(x)=\prod_{i=1}^{n}\left\langle x, v_{i}\right\rangle$.

Proof. Let's denote by $A$ the $n \times n$ matrix $A=\left(v_{1}, \cdots, v_{n}\right)$ consisting of row vectors $v_{1}, \cdots, v_{n}$. Then, we have $A^{t} e_{i}=v_{i}, i=1, \cdots, n$, where $A^{t}$ is the transpose of the matrix $A$ and $\left\{e_{i}\right\}_{i=1}^{n}$ the natural basis of $\mathbb{E}^{n}$.

Now, we consider the linear transformation $T$ of the Euclidean space $\mathbb{E}^{n}$ defined by $y=B x$, where $B=\alpha A$ with $\alpha=(|\operatorname{det} A|)^{-1 / n}$. Then, the transformation $T$ is volume preserving. Since $\left\langle y, e_{i}\right\rangle=\alpha\left\langle x, v_{i}\right\rangle, i=$ $1, \cdots, n, T$ transforms each hyperplanes $P_{i}:\left\langle x, v_{i}\right\rangle=0, i=1, \cdots, n$ to the coordinate hyperplanes $\left\langle y, e_{i}\right\rangle=0$ and the cone $C:\left\langle x, v_{i}\right\rangle>0, i=$ $1, \cdots, n$ to the cone $C^{\prime}:\left\langle y, e_{i}\right\rangle>0, i=1, \cdots, n$.

Let's put $M^{\prime}=T(M)$ and $p^{\prime}=T(p)$, respectively. Since the tangent hyperplane $P$ of $M$ at $p$ is transformed to the tangent hyperplane $P^{\prime}$ of $M^{\prime}$ at $p^{\prime}$, the volume $V\left(p^{\prime}\right)$ of the region of the cone $C^{\prime}$ cut off by the hyperplane $P^{\prime}$ is given by

$$
V\left(p^{\prime}\right)=V(p), \quad p \in M
$$

Since $V(p)$ is independent of the point $p \in M, V\left(p^{\prime}\right)$ is independent of the point $p^{\prime} \in M^{\prime}$. Thus, Proposition 2.2 completes the proof.

For each $i=1, \cdots, n$, we denote by $d_{i}(p)$ the distance from the point $p \in M$ to the hyperplane $P_{i}:\left\langle x, v_{i}\right\rangle=0$. Let's put $d(p)=\prod_{i=1}^{n} d_{i}(p)$. Then we have

Remark 2.4. The conditions in Theorem 2.3 is equivalent to the following. 
$(D)$ : The function $d(p)$ is a positive constant on $M$.

\section{Elliptic cones}

In this section, first of all, we consider the circular cone in the $n$ dimensional Euclidean space $\mathbb{E}^{n}$ which is defined as follows:

$$
C: x_{n}^{2}>|x|^{2}, x=\left(x_{1}, \cdots, x_{n-1}\right), x_{n}>0 .
$$

If we use the Lorentzian scalar product $\langle\cdot, \cdot\rangle$, which is defined by

$$
\langle a, b\rangle_{1}=\sum_{i=1}^{n-1} a_{i} b_{i}-a_{n} b_{n}
$$

for $a, b \in \mathbb{E}^{n}$, then the circular cone is given by $C:\langle x, x\rangle_{1}<0, x_{n}>0$.

Now, we obtain the following.

Lemma 3.1. Suppose that $M$ is a strictly convex hypersurface in the $n$-dimensional Euclidean space $\mathbb{E}^{n}$ which lies in the circular cone $C$. If we denote by $Z(p)$ a nonzero normal to the hypersurface $M$ at $p \in M$, then the volume $V(p)$ is given by

$$
V(p)=\frac{\omega_{n-1}}{n} \frac{|\langle p, Z(p)\rangle|^{n}}{\left|\langle Z(p), Z(p)\rangle_{1}\right|^{n / 2}},
$$

where $\langle Z(p), Z(p)\rangle_{1}<0$ and $\omega_{n-1}$ denotes the volume of $(n-1)$ dimensional ball.

Proof. First of all, we find a transformation $T: y=A x$ of the Euclidean space $\mathbb{E}^{n}$ which takes the cone $C$ to itself and the tangent hyperplane $P$ at $p \in M$ to a hyperplane $P^{\prime}$ which is perpendicular to the $y_{n}$-axis.

Since the volume $V(p)$ is finite, we assume that $\langle Z(p), Z(p)\rangle_{1}<0$. For simplicity, we put $a=\left(a_{1}, \cdots, a_{n}\right)=Z(p) / \sqrt{\left|\langle Z(p), Z(p)\rangle_{1}\right|}$. Then we have $\langle a, a\rangle_{1}=-1$. The tangent hyperplane $P$ of $M$ at $p$ is given by

$$
P:\langle x-p, a\rangle=0 .
$$

Or, equivalently, it can be written as

$$
P:\langle x-p, \tilde{a}\rangle_{1}=0,
$$

where $\tilde{a}=\left(a_{1}, \cdots, a_{n-1},-a_{n}\right)$. Note that $\langle\tilde{a}, \tilde{a}\rangle_{1}=-1$. 
Consider the matrix $B=\left(u_{1}, u_{2}, \cdots, u_{n-1}, \tilde{a}\right)$ consisting of $n$ column vectors satisfying

$$
\left\langle u_{i}, \tilde{a}\right\rangle_{1}=0, \quad\left\langle u_{i}, u_{j}\right\rangle_{1}=\delta_{i j}, \quad i, j=1,2, \cdots n-1 .
$$

It is always possible to choose $u_{1}, u_{2}, \cdots, u_{n-1}$ satisfying (3.5). In fact, they form an orthonormal basis of the orthogonal complement of the time-like unit vector $\tilde{a}$ with respect to the Lorentzian scalar product. It is obvious that $\operatorname{det} B= \pm 1$.

For the matrix $A=B^{-1}$, we show that the transformation $T: y=A x$ has the desired properties as follows.

If $y=A x$, then we have

$$
x=B y=y_{1} u_{1}+\cdots+y_{n-1} u_{n-1}+y_{n} \tilde{a} .
$$

Hence, we get from (3.5)

$$
\langle x, x\rangle_{1}=\langle B y, B y\rangle_{1}=\langle y, y\rangle_{1},
$$

which shows that the transformation $T$ preserves the Lorentzian scalar product and hence preserves the cone $C$.

It follows from (3.7) that by the transformation $T$, the tangent hyperplane $P$ of $M$ at $p$ is transformed to the following hyperplane $P^{\prime}$

$$
P^{\prime}:\left\langle y-p^{\prime}, e_{n}\right\rangle_{1}=0,
$$

where $p^{\prime}=A p, e_{n}=(0, \cdots, 0,1)$ and we used $e_{n}=A \tilde{a}$. Furthermore, (3.7) shows that

$$
\left\langle p^{\prime}, e_{n}\right\rangle_{1}=\langle A p, A \tilde{a}\rangle_{1}=\langle p, \tilde{a}\rangle_{1}=\langle p, a\rangle,
$$

which, together with (3.8) implies that the hyperplane $P^{\prime}$ is given by

$$
P^{\prime}:\left\langle y, e_{n}\right\rangle=-\langle p, a\rangle .
$$

This shows that the transformation $T: y=A x$ has the desired properties.

Summarizing the above, we see that the transformation $T$ takes the region of the cone $C$ cut off by the tangent hyperplane $P$ of $M$ at $p$ to the region of the cone $C$ cut off by the hyperplane $P^{\prime}$ which is perpendicular to the $y_{n}$-axis.

Hence, it follows from (3.10) that the volume $V(p)$ is given by

$$
V(p)=\frac{\omega_{n-1}}{n|\operatorname{det}(A)|}|\langle p, a\rangle|^{n}=\frac{\omega_{n-1}}{n}|\langle p, a\rangle|^{n},
$$

where $\omega_{n-1}$ denotes the volume of $(n-1)$-dimensional ball. Thus we get

$$
V(p)=\frac{\omega_{n-1}}{n} \mid \frac{|\langle p, Z(p)\rangle|^{n}}{\left|\langle Z(p), Z(p)\rangle_{1}\right|^{n / 2}} .
$$


This completes the proof.

Example 3.2. Consider the elliptic hyperboloid $g^{-1}(k)$ with $k<0$ which is given by

$$
g\left(x_{1}, x_{2}, \cdots, x_{n}\right)=\langle x, x\rangle_{1}, \quad x_{n}>0 .
$$

Then the elliptic hyperboloid $g^{-1}(k)$ with $k<0$ is contained in the circular cone $C:\langle x, x\rangle_{1}<0, x_{n}>0$.

For $p=\left(x_{1}, \cdots, x_{n}\right) \in g^{-1}(k)$ and

$$
Z(p)=\nabla g(x) / 2=\left(x_{1}, \cdots, x_{n-1},-x_{n}\right),
$$

we have

$$
\langle p, Z(p)\rangle=\langle Z(p), Z(p)\rangle_{1}=k .
$$

Hence, we get from (3.12) that

$$
V(p)=\frac{\omega_{n-1}}{n}|k|^{n / 2},
$$

which is independent of the point $p \in g^{-1}(k)$.

Next, we prove

Proposition 3.3. Let $M$ be a smooth strictly convex hypersurface in the $n$-dimensional Euclidean space $\mathbb{E}^{n}$ which lies in the circular cone $C:\langle x, x\rangle_{1}<0, x_{n}>0$. Suppose that the volume $V(p)$ of the region of the cone $C$ cut off by the tangent hyperplane $P$ of $M$ at $p$ is a positive constant on $M$. Then $M$ is part of an elliptic hyperboloid $g^{-1}(k)$ with $k<0$ which is defined by

$$
g(x)=\langle x, x\rangle_{1}, \quad x_{n}>0 .
$$

Proof. For a fixed point $p_{0}$ on the hypersurface $M$, we may assume that a neighborhood $U$ of $p_{0} \in M$ is the graph of a function $x_{n}=f(x), x=$ $\left(x_{1}, \cdots, x_{n-1}\right)$ defined on an open set $O$ in the Euclidean space $\mathbb{E}^{n-1}$.

We consider the nonzero normal $Z(p)$ to $M$ at $p=(x, f(x))$ given by

$$
Z(p)=\left(-f_{1}(x), \cdots,-f_{n-1}(x), 1\right),
$$

where $f_{i}$ denotes the partial derivative of $f$ with respect to $x_{i}, i=$ $1, \cdots, n-1$. Then, Lemma 3.1 shows that for the constant $a= \pm\left(n / \omega_{n-1}\right)^{1 / n}$ the volume function $V(p)$ is given by

$$
a V(p)^{2 / n}=\alpha(x)^{2} \beta(x)^{-1},
$$

on the open set $O_{1}$ defined by $O_{1}=\{x \in O \mid \beta(x)>0\}$, where

$$
\alpha(x)=f(x)-\langle x, \nabla f(x)\rangle
$$


and

$$
\beta(x)=1-\sum_{j=1}^{n-1} f_{j}(x)^{2} .
$$

Henceforth, we proceed on the open set $O_{1}$. By differentiating (3.15) with respect to $x_{i}, i=1, \cdots, n-1$, it is straightforward to show that for each $i=1, \cdots, n-1$

$$
\frac{\partial}{\partial x_{i}}\left\{a V(p)^{2 / n}\right\}=2 \alpha(x) \beta(x)^{-2}\left\{\sum_{j=1}^{n-1} f_{i j}(x)\left(\alpha(x) f_{j}(x)-\beta(x) x_{j}\right)\right\} .
$$

Now, suppose that the volume function $V(p)$ is a positive constant on the hypersurface $M$. Then, (3.18) implies

$$
\alpha(x) \beta(x)^{-2}\left\{\sum_{j=1}^{n-1} f_{i j}(x) A_{j}(x)\right\}=0,
$$

where we denote

$$
A_{j}(x)=\alpha(x) f_{j}(x)-\beta(x) x_{j} .
$$

It follows from (3.19) that for each $i=1, \cdots, n-1$ we have

$$
\sum_{j=1}^{n-1} f_{i j}(x) A_{j}(x)=0 \text {. }
$$

Since the hypersurface $M$ is strictly convex, the Hessian matrix $\left(f_{i j}(x)\right)$ is nonsingular ([6, p.93]). Hence, together with (3.20), (3.21) shows that for each $j=1, \cdots, n-1$

$$
\alpha(x) f_{j}(x)-\beta(x) x_{j}=0 .
$$

We now differentiate $\alpha$ and $\beta$ in (3.16) and (3.17) with respect to $x_{i}, i=1,2, \cdots, n-1$, respectively. Then, we obtain for $i=1,2, \cdots, n-1$

$$
\alpha_{i}=-\sum_{j=1}^{n-1} x_{j} f_{i j}(x), \quad \beta_{i}=-2 \sum_{j=1}^{n-1} f_{j}(x) f_{i j}(x) .
$$

Hence, using (3.22), we get for $i=1,2, \cdots, n-1$

$$
2 \beta \alpha_{i}=\alpha \beta_{i},
$$

which shows that for some nonzero constant $c$

$$
\beta(x)=c \alpha(x)^{2} .
$$


Substituting (3.25) into (3.22) implies for each $j=1,2, \cdots, n-1$

$$
f_{j}=c \alpha(x) x_{j} .
$$

Together with (3.26), (3.16) shows

$$
\alpha\left\{1+c \sum_{j=1}^{n-1} x_{j}^{2}\right\}=f(x) .
$$

Hence, by combining (3.26) and (3.27), we get for each $j=1,2, \cdots, n-1$

$$
f_{j}\left\{1+c \sum_{k=1}^{n-1} x_{k}^{2}\right\}=c x_{j} f(x) .
$$

Thus, integrating (3.28) implies that for some positive constant $d$

$$
f(x)=d \sqrt{1+c \sum_{k=1}^{n-1} x_{k}^{2}} .
$$

That is, on the open set $O_{1}, f(x)$ is of the following form

$$
f(x)=\sqrt{a|x|^{2}+b},
$$

where $a \neq 0$ and $b>0$.

Now, we check the volume $V(p)$ as follows. For a point $p=(x, f(x)) \in$ $U$ with $x \in O_{1}$, we consider a normal vector $Z(p)=\left(-a x_{1}, \cdots,-a x_{n-1}\right.$, $f(x))$. Then we have

$$
\langle p, Z(p)\rangle=b \quad \text { and } \quad\langle Z(p), Z(p)\rangle_{1}=a(a-1)|x|^{2}-b .
$$

Hence, Lemma 3.1 shows that $a$ must be 1 .

Together with (3.30), (3.31) implies that on the open set $O_{1}=\{x \in$ $O \mid \beta(x)>0\}, f(x)$ is given by

$$
f(x)=\sqrt{|x|^{2}+b} .
$$

Since $\beta(x)=b / f(x)^{2}>0$ on the whole domain of $f(x)$, we see that $O=O_{1}$. Thus, every point $p=\left(x_{1}, \cdots, x_{n}\right)$ on the neighborhood $U$ of $p_{0}$ satisfies

$$
x_{1}^{2}+\cdots+x_{n-1}^{2}-x_{n}^{2}=-b, \quad x_{n}>0 .
$$

By the connectedness of the hypersurface $M$, we conclude that $M$ is part of the level hypersurface $g^{-1}(k)$ with $k<0$ of the function

$$
g(x)=\langle x, x\rangle_{1}, \quad x_{n}>0 .
$$

This completes the proof. 
We now consider the elliptic cone $C: x_{n}^{2}>a_{1}^{2} x_{1}^{2}+\cdots+a_{n-1}^{2} x_{n-1}^{2}, x_{n}>$ 0 , where $a_{i}>0$ for $i=1, \cdots, n-1$. Let $M \subset C$ be a smooth strictly convex hypersurface in the $n$-dimensional Euclidean space $\mathbb{E}^{n}$.

Theorem 3.4. For the elliptic cone $C$, suppose that $M \subset C$ is a smooth strictly convex hypersurface. Then, the following are equivalent.

1) The volume $V(p)$ is a positive constant on $M$.

2) The hypersurface $M$ is part of an elliptic hyperboloid $g^{-1}(k)$ with $k<0$ which is defined by

$$
g\left(x_{1}, x_{2}, \cdots, x_{n}\right)=a_{1}^{2} x_{1}^{2}+\cdots+a_{n-1}^{2} x_{n-1}^{2}-x_{n}^{2}, \quad x_{n}>0 .
$$

Proof. We consider the linear transformation $T$ of the Euclidean space $\mathbb{E}^{n}$ defined by $y_{1}=a_{1} x_{1}, \cdots, y_{n-1}=a_{n-1} x_{n-1}, y_{n}=x_{n}$. Then, the transformation $T$ takes the cone $C$ to the circular cone $C^{\prime}: y_{n}^{2}>$ $|y|^{2}, y_{n}>0$, the hypersurface $M \subset C$ to the hypersurface $M^{\prime} \subset C^{\prime}$, $p \in M$ to the corresponding point $p^{\prime} \in M^{\prime}$ and the tangent hyperplane $P$ of $M$ at $p$ to the tangent hyperplane $P^{\prime}$ of $M^{\prime}$ at $p^{\prime}$. Hence the region of the cone $C$ cut off by the tangent hyperplane $P$ of $M$ at $p$ is transformed to the corresponding region of the cone $C^{\prime}$. Thus, the volume $V\left(p^{\prime}\right)$ of the corresponding region of the cone $C^{\prime}$ is given by

$$
V\left(p^{\prime}\right)=a_{1} \cdots a_{n-1} V(p), \quad p \in M .
$$

Since $V(p)$ is independent of the point $p \in M, V\left(p^{\prime}\right)$ is independent of the point $p^{\prime} \in M$. Thus, together with Example 3.2, Proposition 3.3 shows that 1$) \Leftrightarrow 2$ ).

This completes the proof.

\section{References}

[1] Kim, D.-S. and Kim, Y. H., A characterization of ellipses, Amer. Math. Monthly 114 (2007), no. 1, 66-70.

[2] Kim, D.-S. and Kim, Y. H., Some characterizations of spheres and elliptic paraboloids, Linear Algebra Appl. 437 (2012), no. 1, 113-120.

[3] Kim, D.-S. and Kim, Y. H., Some characterizations of spheres and elliptic paraboloids II, Linear Algebra Appl., 438 (2013), no. 3, 1356-1364.

[4] Kim, D.-S., Ellipsoids and elliptic hyperboloids in the Euclidean space $\mathbb{E}^{n+1}$, Submitted.

[5] O'Neill, B., Elementary differential geometry, Revised second edition, Elsevier/Academic Press, Amsterdam, 2006. 
Dong-Soo Kim

Department of Mathematics, Chonnam National University, Kwangju 500-757, Korea.

E-mail: dosokim@chonnam.ac.kr

\section{Booseon Song}

Department of Mathematics, Chonnam National University, Kwangju 500-757, Korea.

E-mail: booseons@gmail.com 